Research Article

\title{
Electromagnetic Nondestructive Testing by Perturbation Homotopy Method
}

\author{
Liang Ding ${ }^{1}$ and Jun Cao ${ }^{2}$ \\ ${ }^{1}$ Department of Mathematics, Northeast Forestry University, No. 26 Hexing Road, Xiangfang District, Harbin 150040, China \\ ${ }^{2}$ College of Mechanical and Electrical Engineering, Northeast Forestry University, No. 26 Hexing Road, Xiangfang District, \\ Harbin 150040, China
}

Correspondence should be addressed to Jun Cao; nefuding@gmail.com

Received 23 September 2013; Accepted 2 January 2014; Published 5 March 2014

Academic Editor: Manuel Ruiz-Galan

Copyright (C) 2014 L. Ding and J. Cao. This is an open access article distributed under the Creative Commons Attribution License, which permits unrestricted use, distribution, and reproduction in any medium, provided the original work is properly cited.

\begin{abstract}
Now electromagnetic nondestructive testing methods have been applied to many fields of engineering. But traditional electromagnetic methods (usually based on least square and local iteration) just roughly give the information of location, scale, and quality. In this paper we consider inverse electromagnetic problem which is concerned with the estimation of electric conductivity of Maxwell's equations (2D and 3D). A perturbation homotopy method combined with damping Gauss-Newton methods is applied to the inverse electromagnetic problem. This method differs from traditional homotopy method. The structure of homotopy function is similar to Tikhonov functional. Sets of solutions are produced by perturbation for every homotopy parameter $\lambda=\lambda_{i}$, $i=0, \ldots, L$. At each iterative step of the algorithm, we add stochastic perturbation to numerical solutions. The previous solution and perturbation solution are regarded as the initial value in the next iteration. Although the number of solution in set increased, it increased the likelihood of obtaining correct solution. Results exhibits clear advantages over damping Gauss-Newton method and testify that it is an available method, especially on aspects of wide convergence and precision.
\end{abstract}

\section{Introduction}

In this paper, we discuss an inverse problem of electromagnetic field. For a given source, the direct problem is to determine the electromagnetic field for the known coefficient, which has been well studied [1]. Our work is devoted to the numerical solution of the electrical conductivity inversion. This problem is a model problem for many real industrial applications including mathematical physics, atmospheric science, quantum mechanics, telemetry, nondestructive testing, and medical imaging. The first pioneering solution of the fully 3D Maxwell's equation inverse problem was presented by Eaton more than 15 years ago [2]. Yet in spite of this, until recently, the trial-and-error forward modelling was almost the only available tool to interpret the fully 3D EM dataset. Today the situation has slightly improved, and the methods of unconstrained nonlinear optimization [3] are gaining popularity to address the problem. Although many new theoretical approaches have been applied to this domain $[4,5]$ successfully and the high level of computer hardware is changing with each passing day, the desired numerical solution of the inverse electromagnetic problems is still difficult to resolve for the following reasons. (1) The rapid, accurate, and stable forward numerical solution [6-9] is generally required by inverse problem; it may accelerate convergence of the inverse problem (especially, for models with low conductivity contrasts) [10,11], but the general stable and accurate forward solution is still an open problem. (2) The problem has the property of ill-posedness and nonlinearity. It means that the solution is not unique; any slight noise will lead to huge error. (3) In order to get a unique solution which is dependent on the measured data continuously and stably, it is necessary to investigate a stable regularization theory. Therefore, it is meaningful to design a highly efficient, numerically stable, and globally convergent algorithm to overcome the abovementioned difficulties.

Homotopy method is a global convergence algorithm for nonlinear operator equation. It has been applied to inverse problem in recent decades. Han et al. first applied homotopy method to inverse petroleum well-logging problem 


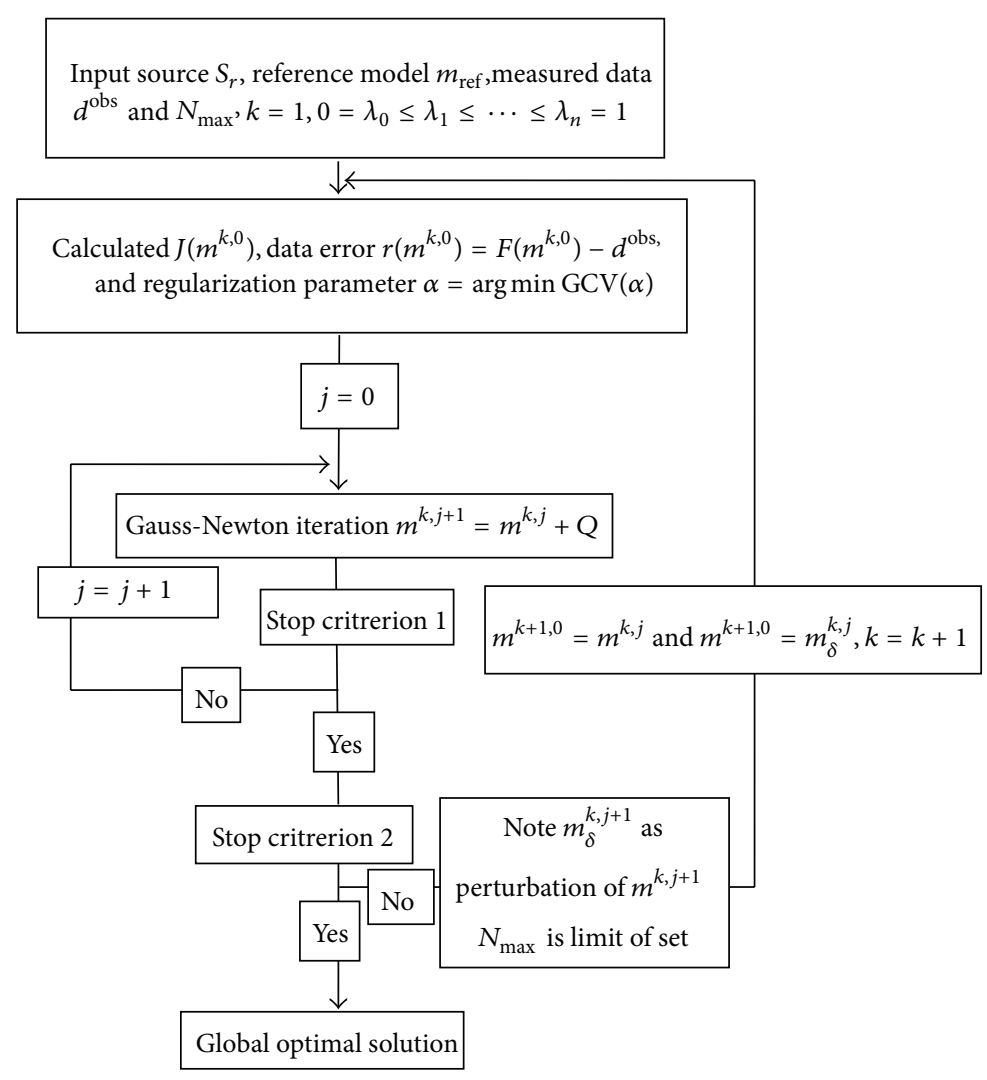

FIGURE 1: The sketch map of perturbation homotopy method.

in 1991 [12]. Afterwards, they extended this method to general parameter identification. Fu et al. gave the numerical solution of inverse acoustic problem and elastic wave equation with fluid saturated porous media by homotopy method, respectively $[13,14]$. Vasco applied homotopy method to geophysics inverse problem in 1994 [15]. He makes use of singularity and bifurcation theory to research inverse problem. Dunlavy applied homotopy optimization method to protein structure prediction in 2005 [16]. In summary, the homotopy method has shown great advantage for solving inverse problem, especially in aspects of enlarging the convergence domain and enhancing stability with noise. But homotopy inversion method is still far away from perfection and bifurcation, and convolution about homotopy curves will appear in the process of computation.

As mentioned above, we will introduce a perturbation homotopy method for inverse electromagnetic problem. The model is Maxwell's equations. In each iterative step of the algorithm, we add stochastic perturbation to numerical solutions. The previous solution and perturbation solution are regarded as the initial value in the next iteration. Although the number of solution in set increased, it increased the likelihood of obtaining correct solution. With the number of elements in set increasing, we require the input value $N_{\max }$ (maximum number of solutions in a set) to limit the size of set.

The paper is organized as follows. We first briefly review some basic ideas about inverse electromagnetic problem and homotopy. In Section 2, we develop perturbation homotopy method and derive the basic coefficient identification algorithm using this method. In Section 3, numerical experiments are presented which demonstrate the performance of the algorithm and indicate the validity of this method. And finally, in Section 4, some conclusions and future directions are given.

\section{Electromagnetic Forward Model}

The time domain Maxwell equations has the form

$$
\begin{gathered}
\nabla \times \mathbf{E}+\mu \frac{\partial \mathbf{H}}{\partial t}=0 \\
\nabla \times \mathbf{H}-\sigma \mathbf{E}-\varepsilon \frac{\partial \mathbf{E}}{\partial t}=s_{r}(t),
\end{gathered}
$$

over the domain $\Omega \times[0, T]$, where $\mathbf{E}$ and $\mathbf{H}$ are the electric and magnetic fields, $\sigma, \varepsilon$, and $\mu$ are conductivity, dielectric constant, and permeability, respectively, and $s_{r}(t)$ is transmitting source. Discretizing (1) over time grid $\left[t_{n}, t_{n+1}\right]$, we get the equations

$$
\begin{gathered}
\nabla \times \mathbf{E}^{n+1}+\tau_{n} \mu \mathbf{H}^{n+1}=\tau_{n} \mu \mathbf{H}^{n} \quad \text { in } \Omega, \\
\nabla \times \mathbf{H}^{n+1}-\left(\sigma+\tau_{n} \varepsilon\right) \mathbf{E}^{n+1}=s_{r}^{n+1}-\tau_{n} \varepsilon \mathbf{E}^{n} \quad \text { in } \Omega,
\end{gathered}
$$




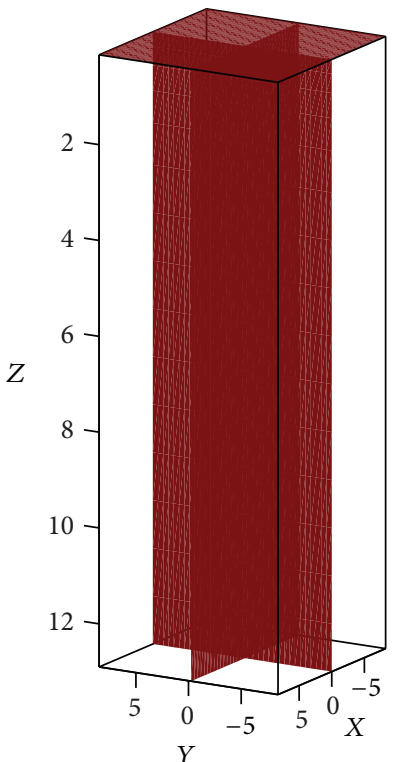

(a)

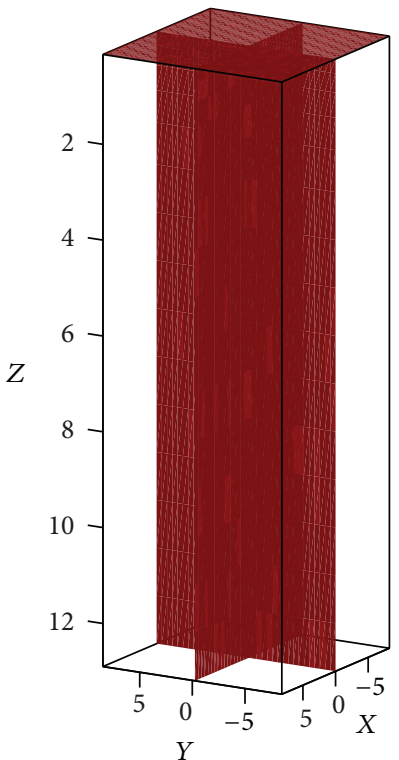

(b)

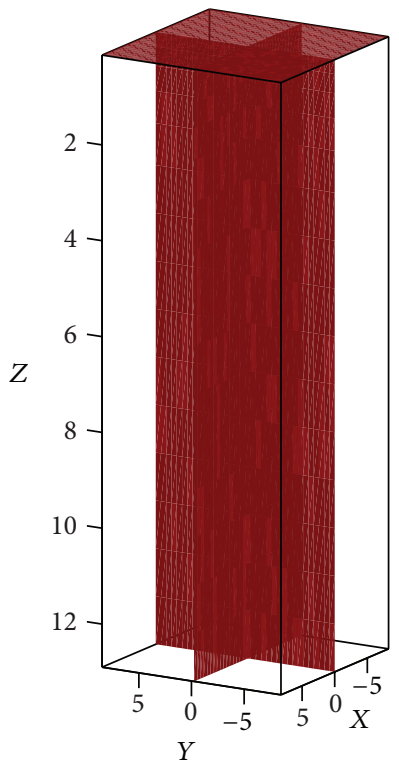

(c)

Figure 2: (a) Original model; (b) inversion results of damping Gauss-Newton; (c) inversion results of perturbation homotopy method.

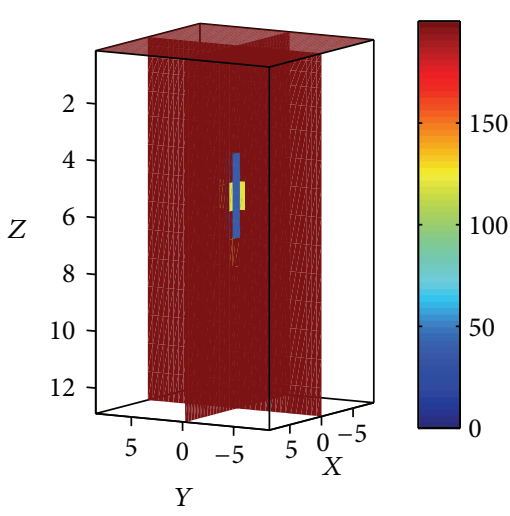

(a)

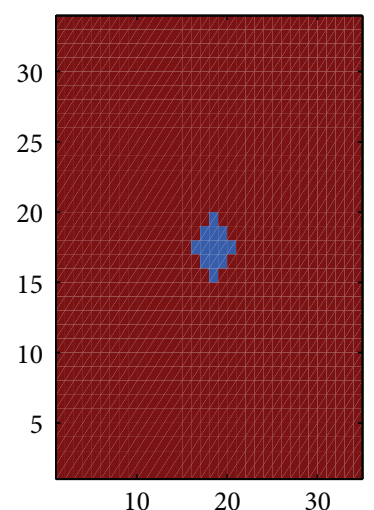

(d)

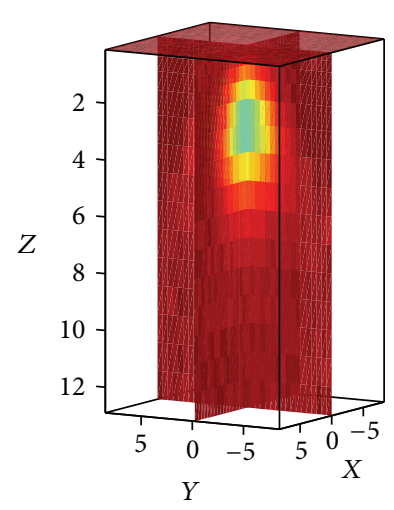

(b)
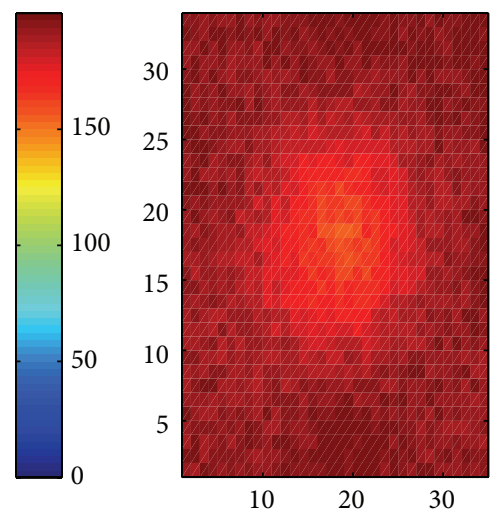

(e)
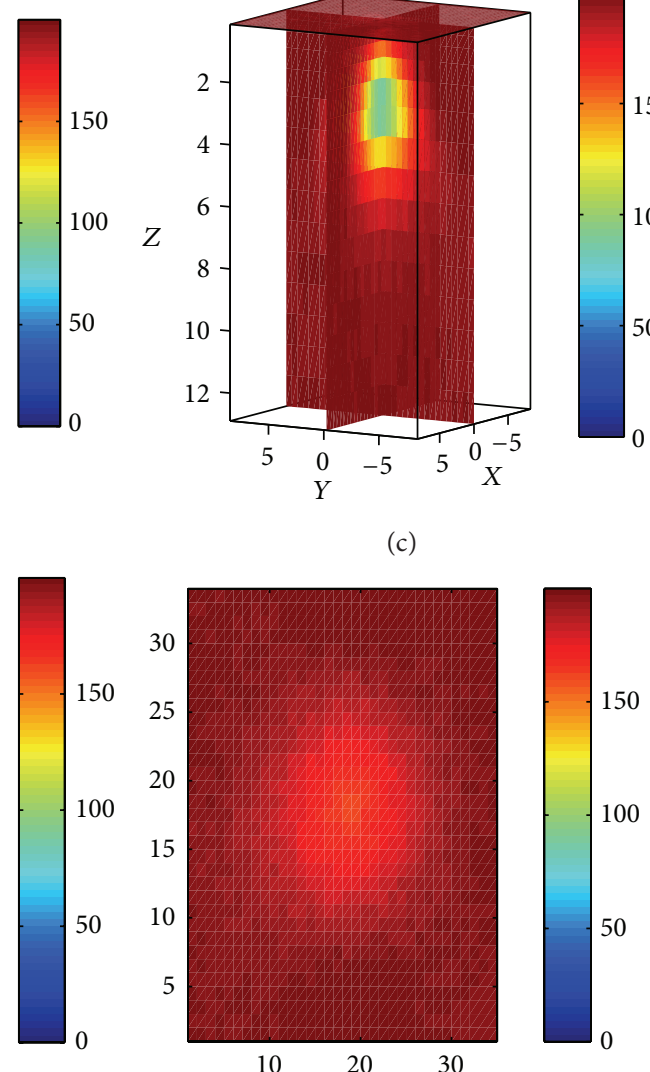

(f)

Figure 3: (a) True model; (b) results of damping Gauss-Newton; (c) results of perturbation homotopy method; (d) cross-section of (a) at $x=0$; (e) cross-section of (b) at $x=0$; (f) cross-section of (c) at $x=0$. 


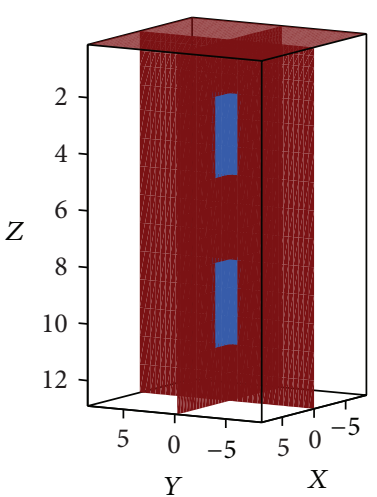

(a)

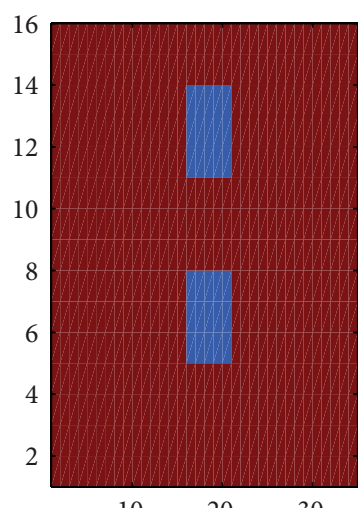

(d)
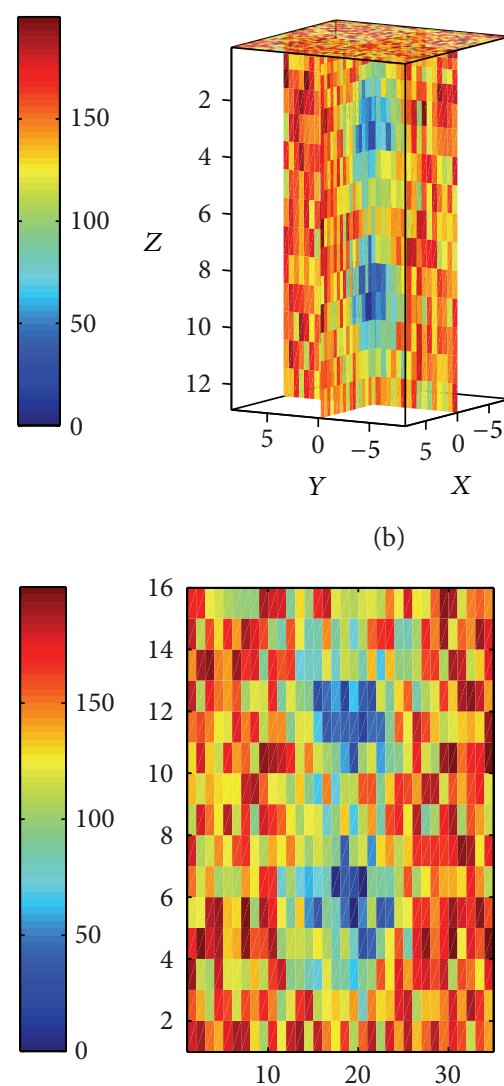

(e)
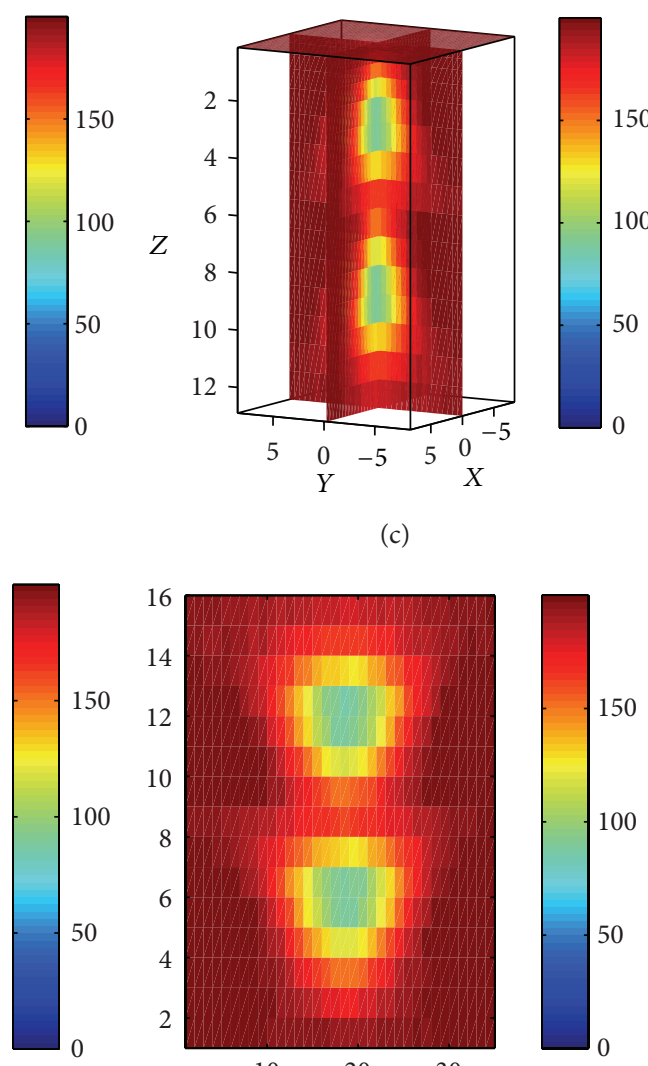

(f)

Figure 4: (a) True model; (b) results of damping Gauss-Newton method; (c) results of perturbation homotopy method; (d) cross-section of (a) at $Z=8$; (e) cross-section of (b) at $Z=8$; (f) cross-section of (c) at $Z=8$.

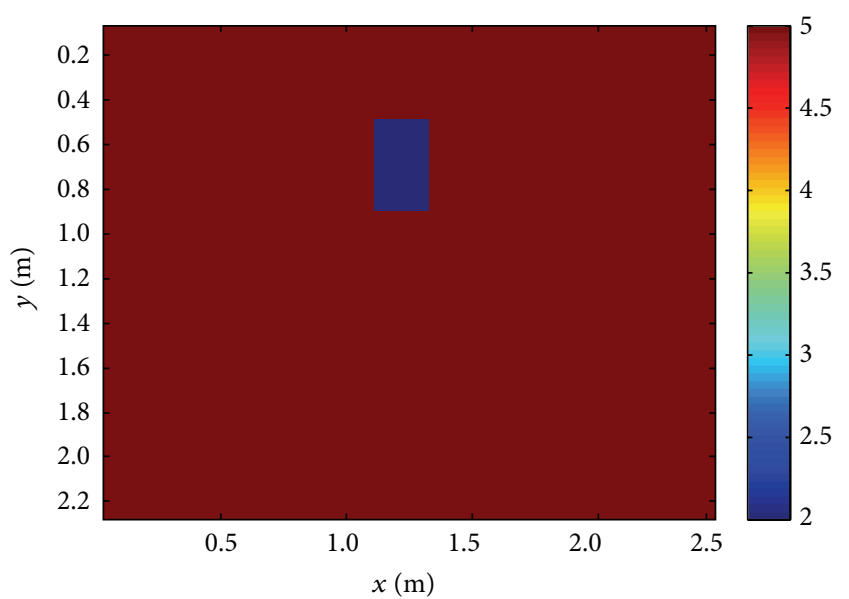

FIGURE 5: Square cavity model.

where $\tau_{n}=1 /\left(t_{n+1}-t_{n}\right)$. With discretization we get matrix equation

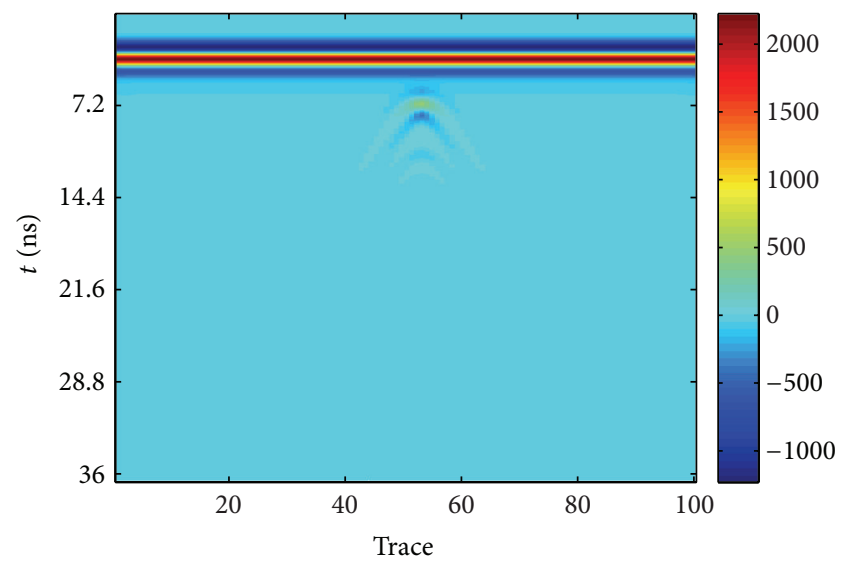

Figure 6: Measured data.
In the inverse problem we want to recover the model $\sigma$ with the measured data. Measured data can be written as
$A(\sigma) u=q$.

$$
d^{\mathrm{obs}}=Q u+\eta
$$




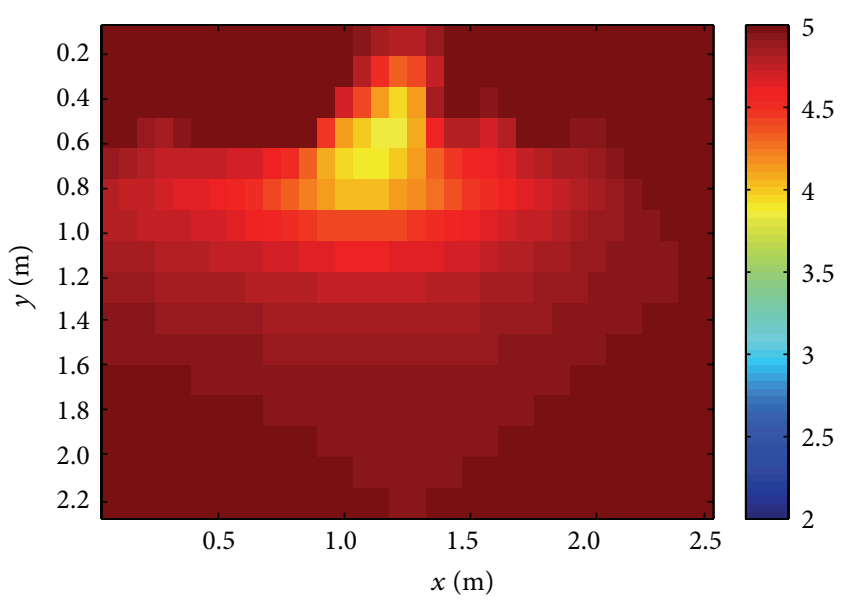

FIgURE 7: Inversion results of Gauss-Newton method.

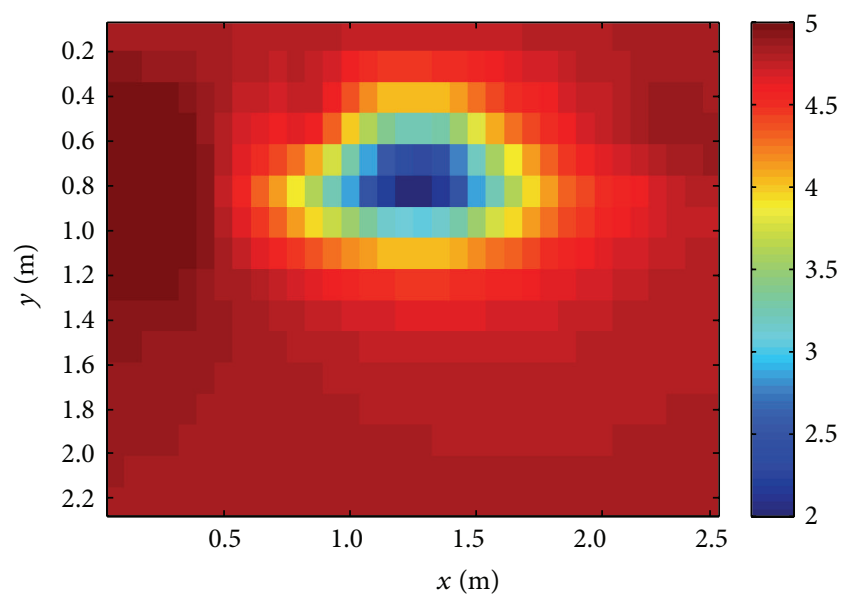

FIGURE 8: Inversion results of perturbation homotopy method.

where $Q$ is a projection operator which projects the electromagnetic fields onto the observation locations and $\eta$ is the measurement noise. From (3) and (4), we can obtain

$$
d^{\text {obs }}=Q A^{-1} q+\eta
$$

\section{Inversion Framework}

3.1. Damping Gauss-Newton Method. For every homotopy parameter $\lambda=\lambda_{i}, i=1, \ldots, L$, we must solve the unconstrained optimization problem

$$
\begin{aligned}
\min _{\sigma} H(\sigma, \lambda)= & \min _{\sigma} \lambda\left\|F(\sigma)-d^{\mathrm{obs}}\right\|^{2} \\
& +(1-\lambda)\left\|W\left(\sigma-\sigma_{\text {ref }}\right)\right\|^{2} .
\end{aligned}
$$

This can be transformed to the Euler-Lagrange system

$$
\begin{aligned}
\frac{\partial H}{\partial \sigma}= & g(\sigma)=(1-\lambda) W^{T} W\left(\sigma-\sigma_{\text {ref }}\right) \\
& +\lambda J(\sigma)^{T}\left(F(\sigma)-d^{\text {obs }}\right) .
\end{aligned}
$$

Denoting $\beta=(1-\lambda) / \lambda$, we obtain

$$
\beta W^{T} W\left(\sigma-\sigma_{\text {ref }}\right)+J(\sigma)^{T}\left(F(\sigma)-d^{\text {obs }}\right)=0,
$$

where $\beta$ played a regularization parameter role. In order to avoid the calculation of the second Fréchet derivative of $F$ to $\sigma, F$ is linearized by

$$
F(\sigma+\delta \sigma)=F(\sigma)+J(\sigma) \delta \sigma+R(\sigma, \delta \sigma)
$$

Then the minimization problem is solved iteratively. At the $k$ th iteration, we solve

$$
\begin{aligned}
& \left(J\left(\sigma_{k}^{T}\right) J\left(\sigma_{k}\right)+\beta W^{T} W\right) \delta \sigma \\
& \quad=J\left(\sigma_{k}^{T}\right)\left(d^{\text {obs }}-F\left(\sigma_{k}\right)+J\left(\sigma_{k}\right)\right)-\beta W^{T} W\left(\sigma_{k}-\sigma_{\text {ref }}\right)
\end{aligned}
$$

to find the perturbation $\delta \sigma$. At each iteration, we have an option of solving directly for a perturbation or solving for an updated model. To formulate the latter option, we write $\sigma_{k+1}=\sigma_{k}+\delta \sigma$ and substitute into (10) to obtain

$$
\begin{aligned}
& \left(J\left(\sigma_{k}^{T}\right) J\left(\sigma_{k}\right)+\beta W^{T} W\right) \sigma_{k+1} \\
& \quad=J\left(\sigma_{k}^{T}\right)\left(d^{\text {obs }}-F\left(\sigma_{k}\right)+J\left(\sigma_{k}\right) \sigma_{k}\right)-\beta W^{T} W \sigma_{\text {ref }} .
\end{aligned}
$$

3.2. A Perturbation Homotopy Method. As above mentioned, we can formulate the inverse electromagnetic problem as the following nonlinear operator equation:

$$
F(\sigma)=d^{\text {obs }}
$$

where $F=Q A^{-1} q$ is the forward nonlinear operator. It is very difficult to estimate $\sigma$ in the problem (12) due to the presence of local minima. Therefore we design a globally convergent algorithm which can overcome the local minima, namely, perturbation homotopy method, to solve the nonlinear equation (12). For inverse electromagnetic problem, we want to solve the minimization problem. Given $\phi: X \subseteq R^{n} \rightarrow R$, find $\sigma^{*} \in X \subseteq R^{n}$ such that

$$
\phi\left(\sigma^{*}\right)=\min _{\sigma \in X}\left\|F(\sigma)-d^{\text {obs }}\right\|^{2} .
$$

In order to apply homotopy to PDE optimization problem, we denote

$$
\begin{gathered}
\phi^{0}=\left\|W\left(\sigma-\sigma_{\mathrm{ref}}\right)\right\|^{2}, \\
\phi^{1}=\left\|F(\sigma)-d^{\mathrm{obs}}\right\|^{2},
\end{gathered}
$$

where $\|\cdot\|$ is 2 -norm, $W$ is positive weight function, and $\sigma_{\text {ref }}$ is the known reference model. So continuous homotopy functional $H: R^{n+1} \rightarrow R$ can be written as

$$
H(\sigma, \lambda)=\lambda \phi^{1}+(1-\lambda) \phi^{0}
$$

where $\lambda \in[0,1]$ are homotopy parameters, and the homotopy step length $\Delta_{i}, i=0, \ldots, L-1$, is satisfied with $\sum_{i=0}^{L-1} \Delta_{i}=1$. A general sketch of the homotopy algorithm is as follows. 

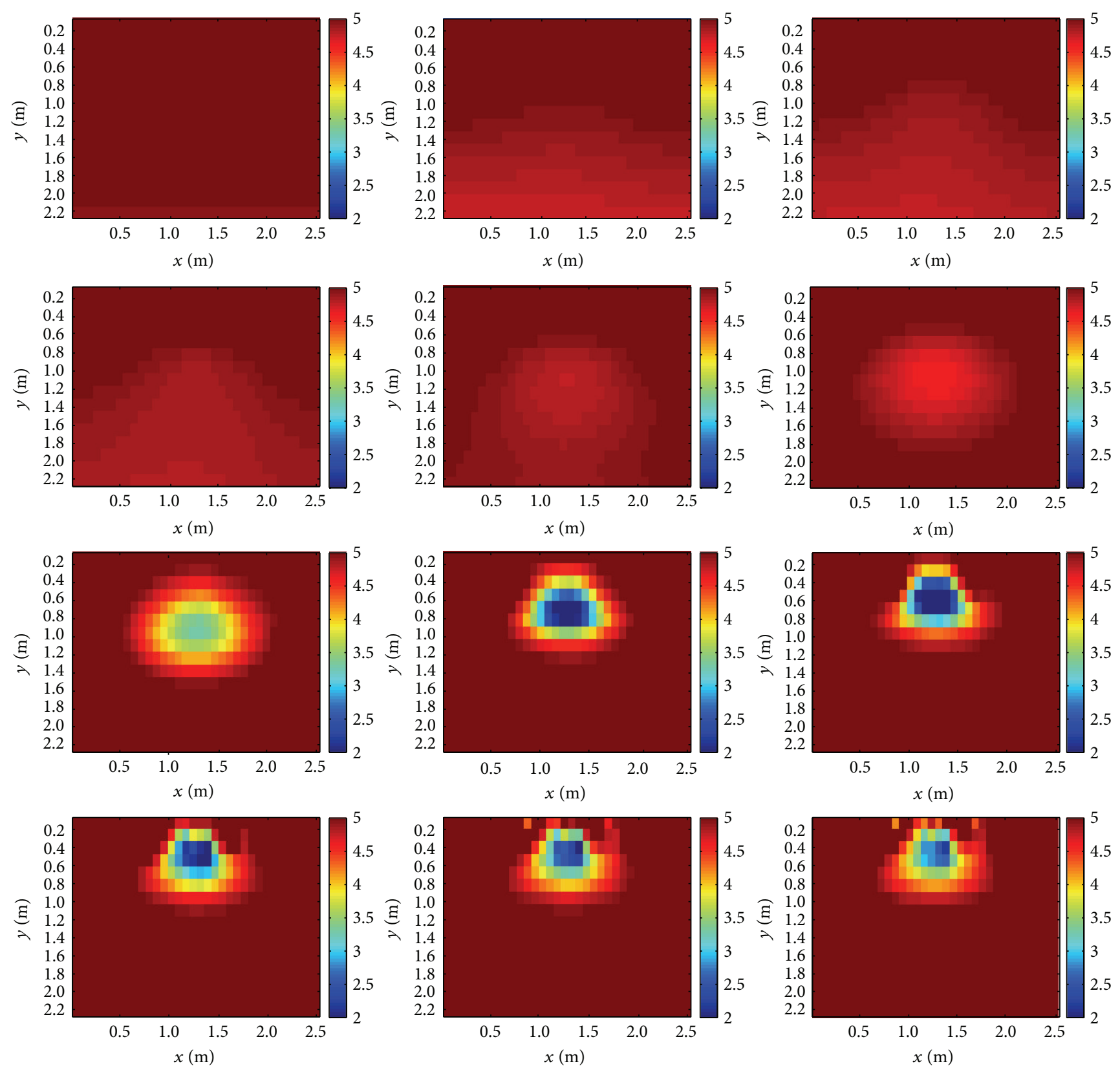

FIGURE 9: Inversion results with different homotopy parameters (1/50001, 1/5001, 1/2001, 1/1501, 1/1001, 1/501, 1/51, 1/6, 2/3, 100/105, 1000/1005, and 100000/100005).

(i) Input: given minimum norm solution of $\phi^{0}$, namely, $\sigma_{0}=\sigma_{\text {ref }}, \lambda_{0}=0$, and $\Delta_{i}>0, i=1, \ldots, L$.

(ii) Update: for $\lambda_{i}=\lambda_{i-1}+\Delta_{i-1}$, we use damping GaussNewton iteration method, compute an approximate solution $\sigma_{i}$ to minimize $\min _{\sigma \in X} H\left(\sigma, \lambda_{i}\right)$ by initial value $\sigma_{i-1}$.

(iii) Output: the iteration will be stopped if the solution $\sigma_{i}$ satisfied stopping criterion; output: $\sigma^{1}=\sigma_{m}$.

The homotopy algorithm's greatest advantage lies in increasing region of convergency. But in the process of computation, the nonconvergent phenomenon often happens to homotopy curve, for example, standstill, convolution, and bifurcation.
These difficulties can make the homotopy method quite complicated. In order to make the homotopy more efficient, sets of solutions are produced by perturbation for every homotopy parameter $\lambda=\lambda_{i}, i=0, \ldots, L$. Although the calculation increased, it ensures the likelihood of obtaining accurate solution. With different homotopy parameter $\lambda_{i}$, the minima is in the sequence, and $\sigma_{i}$ is solved by damping GaussNewton method with the initial value $\sigma_{i-1}$. The next set of local minima $\left(\lambda=\lambda_{i+1}\right)$ is solved with the initial values in the previous set $\left(\lambda=\lambda_{i}\right)$ which has one or more perturbations of each of those points in the set. We denote the perturbation of solution $\operatorname{per}(\sigma)$. The perturbation stochastically disturbs one or more of the solutions $\sigma$ in set. We use a single initial 


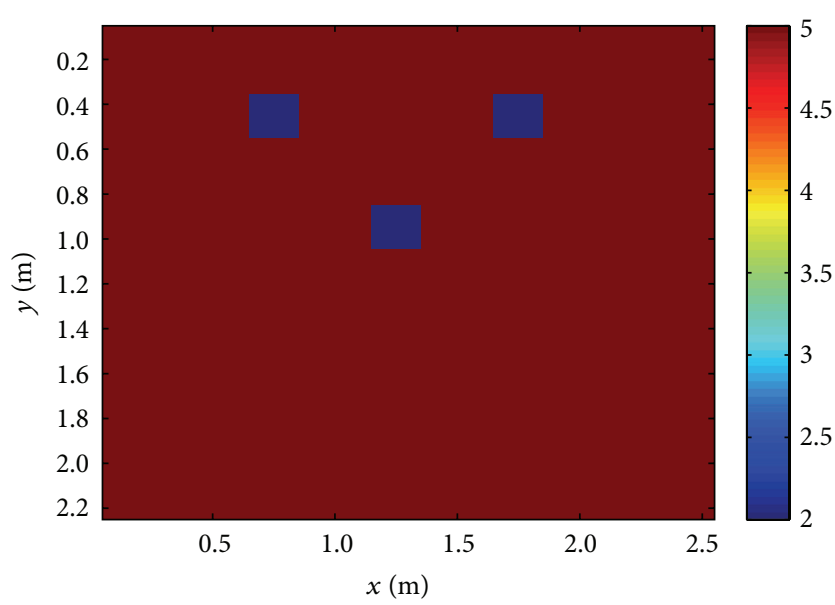

FIgURE 10: Three square cavities model.

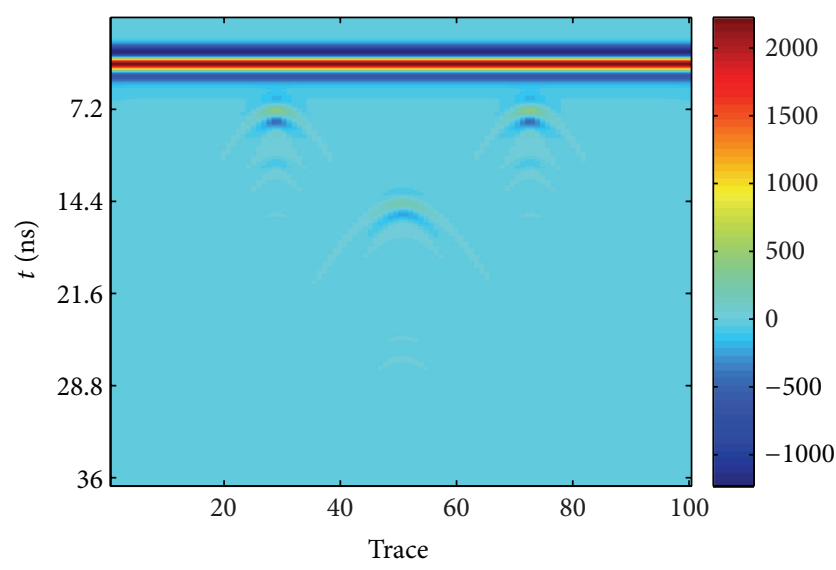

Figure 11: Measured data.

value $\sigma_{0}$ for $\lambda=\lambda_{0}$; the number of solution solved at each homotopy parameter $\lambda$ increases exponentially. Without limit on the size of the set, the number of solutions in the set would be $2^{i}$ when homotopy iteration stops. In order to constraint on computational complexity, it is necessary to limit the size of the set. We denote by $N_{\max }$ the maximum number of solutions in a set. If the number of local minima in the set at the current iteration is less than the maximum set size $N_{\max }$, then every solution in the set is used in the next iteration; otherwise we will choose the preferable solution. An ideal measure of what constitutes the preferable solutions is regularization functional value of (15); solution with the lower regularization functional values is considered the preferable. The perturbation homotopy algorithm is as follows.

(i) Input: given minimum norm solution of $\phi^{0}$, namely, $\sigma_{0}=\sigma_{\text {ref }}, \lambda_{0}=0, N_{\max } \geq 1$, and $\Delta_{i}>0, i=1, \ldots, L$.

(ii) Update: for $\lambda_{i}=\lambda_{i-1}+\Delta_{i-1}$, we use damping Gauss-Newton iteration method, compute an approximate solution $\sigma_{i}^{k}, k=1, \ldots, 2^{i}$, to minimize $\min _{\sigma \in X} H\left(\sigma, \lambda_{i}\right)$ by initial value $\sigma_{i-1}^{j}$ and $\left(\operatorname{per}\left(\sigma_{i-1}\right)\right)^{j}$, $j=1, \ldots, 2^{i}$. (iii) Order the distinct solutions among $\sigma_{i}^{k}, k=1, \ldots, 2^{i}$, from best to worst as $\sigma_{i}^{1}, \sigma_{i}^{2}, \ldots$ and discard any worst solution when the number of elements in $X$ larger than $N_{\max }$.

(iv) Output: the iteration will be stopped if the solution $\sigma_{i}^{m}, 1 \leq m \leq 2^{i}$, is the best solution in the set and satisfied stopping criterion; output: $\sigma^{1}=\sigma_{m}$.

The sketch map of the algorithm is shown in Figure 1.

\section{Numerical Simulation}

In this section, we exhibit clear advantages of perturbation homotopy method over traditional damping Gauss-Newton method in the previous three experiments. In the fourth experiment we analysis the influence of homotopy parameter on inversion results. In the fifth experiment we apply our algorithm to more complicated model and confirm that this algorithm is stable. In the last experiment we solve a simple practice data. In the first three $3 \mathrm{D}$ experiments we select homotopy parameter $\lambda_{i}=i / 10, i=0, \ldots, 10, N_{\max }=2$, and internal Gauss-Newton iteration 5 with each homotopy step in our numerical experiments. The space domain is $\Omega$. The inverse problem is considered on a uniform $32 \times 32 \times 16$ grid. The transmitter source is $s(t)=t^{2} e^{-\alpha t} \sin \left(\omega_{0} t\right)$, where $\alpha=$ $\omega_{0} / \sqrt{3}, \omega_{0}=2 \pi f_{0}$, and $f_{0}=100 \mathrm{MHz}$ is the central frequency. In the last three $2 \mathrm{D}$ experiments $N_{\max }=4$ and internal Gauss-Newton iteration is 10 . The inversion space $\Omega=2.5 \mathrm{~m} \times 2.5 \mathrm{~m}$, space step $\Delta x=\Delta y=0.05 \mathrm{~m}$, sampling time $t=36 \mathrm{~ns}$, time step $\tau=18 \mathrm{~ns}$, and the transmitter source is Ricker wavelet with $900 \mathrm{MHz}$ central frequency.

4.1. Homogeneous Model. We first test a simple homogeneous model, where permittivity $\varepsilon=56064 \times 10^{-12} \mathrm{~F} / \mathrm{m}$, permeability $\mu=0$, and relative conductivity $\sigma=-5.2983$. Both damping Gauss-Newton method and perturbation homotopy method select the same relative initial value $\sigma=-4.8993$. Iterations of damping Gauss-Newton method are five times, elapsed time is 1230.426 seconds, homotopy algorithm's outer iterations $\lambda_{i}, i=1, \ldots, 10$, are ten times, inner local iterations (damping Gauss-Newton method) are three times, and elapsed time is 1405.536 seconds. Figure 2 gives results of true model, damping Gauss-Newton, and perturbation homotopy method. The relative error $\left\|\sigma_{\text {inv }}-\sigma_{\text {true }}\right\| /\left\|\sigma_{\text {true }}\right\|$ of (a) and (b) in Figure 2 is 0.0516 and of (a) and (c) is 0.0830 , where $\sigma_{\text {inv }}$ and $\sigma_{\text {true }}$ are inversion solution and true model, respectively. From Figure 2 we can see that when initial value is close to original conductivity, both methods have satisfactory result, but perturbation homotopy is not more efficient.

4.2. One Circle Cavity Model. The relative conductivity of cavity is -3.2775 ; other parameters have the same value as in the above model. Both methods select the same initial value $\sigma=-1.6094$. Elapsed time of damping Gauss-Newton method and perturbation homotopy method is 11120.536 seconds and 12578.436 seconds, respectively. The relative error $\left\|\sigma_{\text {inv }}-\sigma_{\text {true }}\right\| /\left\|\sigma_{\text {true }}\right\|$ of (a) and (b) in Figure 3 is 

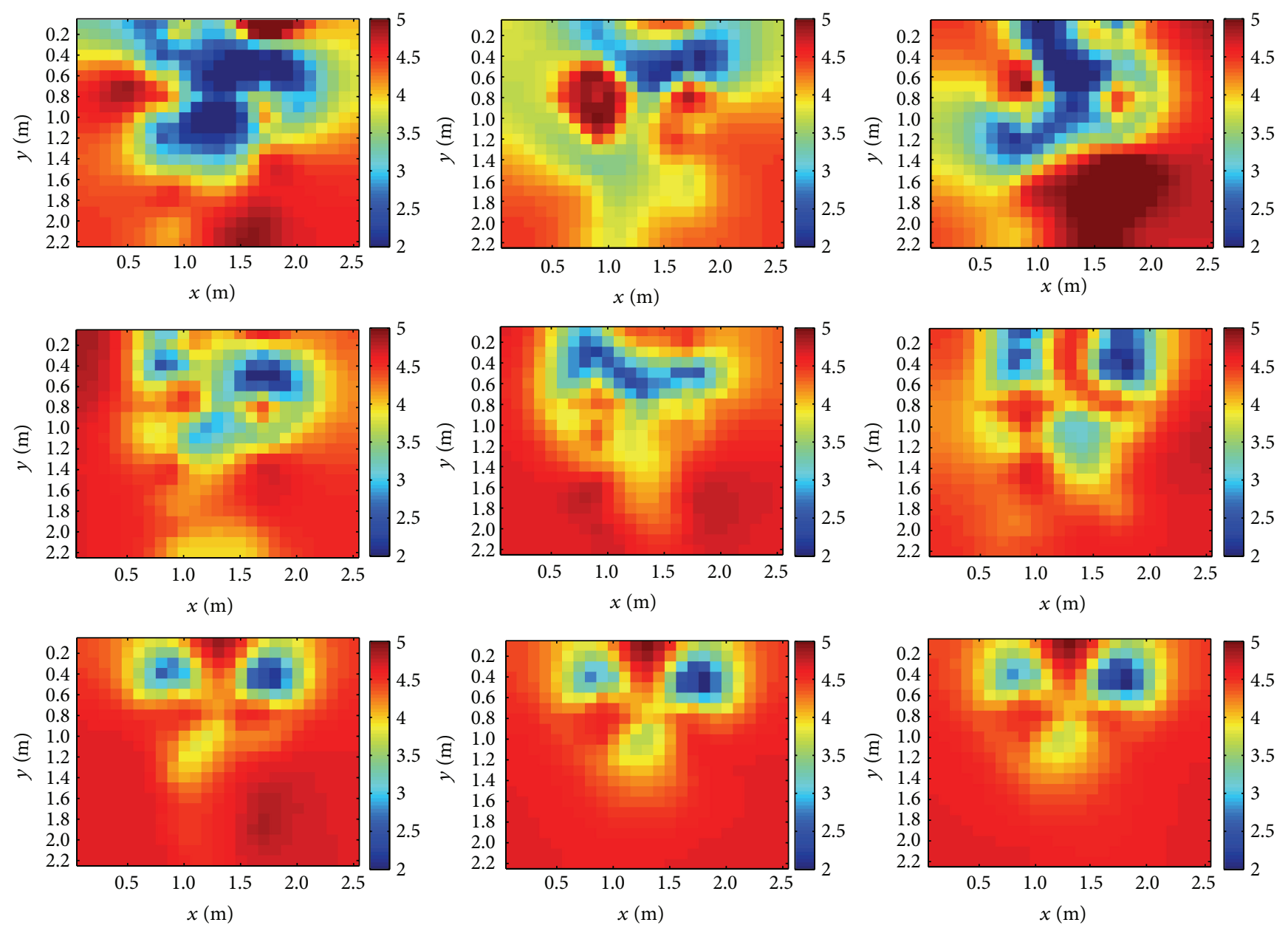

FIGURE 12: Inversion results with different SNR measured data ( $1 \mathrm{~dB}, 3 \mathrm{~dB}, 5 \mathrm{~dB}, 8 \mathrm{~dB}, 10 \mathrm{~dB}, 12 \mathrm{~dB}, 15 \mathrm{~dB}, 20 \mathrm{~dB}$, and $30 \mathrm{~dB})$.

$25.35 \%$ and of (a) and (c) is $9.61 \%$. Although elapsed time of perturbation homotopy is ten times more than damping Gauss-Newton method, when initial value is far away from true value, the latter is inefficient compared to the former.

4.3. Two Cavities Model. The conductivity of two cavities is equal; other parameters have the same value as in Section 4.2. Both methods select the same relative initial value $\sigma=$ -0.8775 . Elapsed time of damping Gauss-Newton method and perturbation homotopy method is 11080.546 seconds and 15458.768 seconds, respectively. Figure 4 gives results of original model, damping Gauss-Newton, and perturbation homotopy method. The relative error $\left\|\sigma_{\text {inv }}-\sigma_{\text {true }}\right\| /\left\|\sigma_{\text {true }}\right\|$ of (a) and (b) in Figure 4 is $49.35 \%$ and of (a) and (c) is $12.85 \%$. When the initial value is further away from original value, damping Gauss-Newton method is invalid.

4.4. One Square Cavity 2D Model. The vertex coordinate of square cavity is $1.2 \mathrm{~m}, 0.4 \mathrm{~m} ; 1.3 \mathrm{~m}, 0.4 \mathrm{~m} ; 1.2 \mathrm{~m}, 0.5 \mathrm{~m} ; 1.3 \mathrm{~m}$, $0.5 \mathrm{~m}$, respectively. The original model structure scheme is shown in Figure 5. Inversion results by Gauss-Newton method are shown in Figure 7. Measured data is shown in Figure 6. With the influence of local minima Figure 7 roughly describes the information of location and scale of square cavity, but the quality information error is huge. Because traditional Tikhonov regularization would smooth the inversion solution, the solution always appears blurring phenomenon at boundary. Inversion results by perturbation homotopy method with different homotopy parameter are shown in Figure 8; the relative error between inversion and original model is $4.55 \%$. We select homotopy parameter as $1 / 50001,1 / 5001,1 / 2001,1 / 1501,1 / 1001,1 / 501,1 / 51,1 / 6,2 / 3$, $100 / 105,1000 / 1005$, and 100000/100005. From Figure 9 we can conclude that the rate of convergence is fast, but when homotopy parameter is close to 1 , numerical solution is diverging, so the optimal solution is not at $\lambda=1$.

4.5. Three Square Cavities 2D Model. The original model structure scheme is shown in Figure 10. Measured data is shown in Figure 11. In order to demonstrate that the method is stable with noise, we add different Gauss white noise to measured data. The SNR (signal-to-noise ratio) is $1 \mathrm{~dB}, 3 \mathrm{~dB}$, $5 \mathrm{~dB}, 8 \mathrm{~dB}, 10 \mathrm{~dB}, 12 \mathrm{~dB}, 15 \mathrm{~dB}, 20 \mathrm{~dB}$, and $30 \mathrm{~dB}$, respectively. From Figure 12 we can see that, with noise decrease, numerical solution is convergent to original model. So our method is stable. 


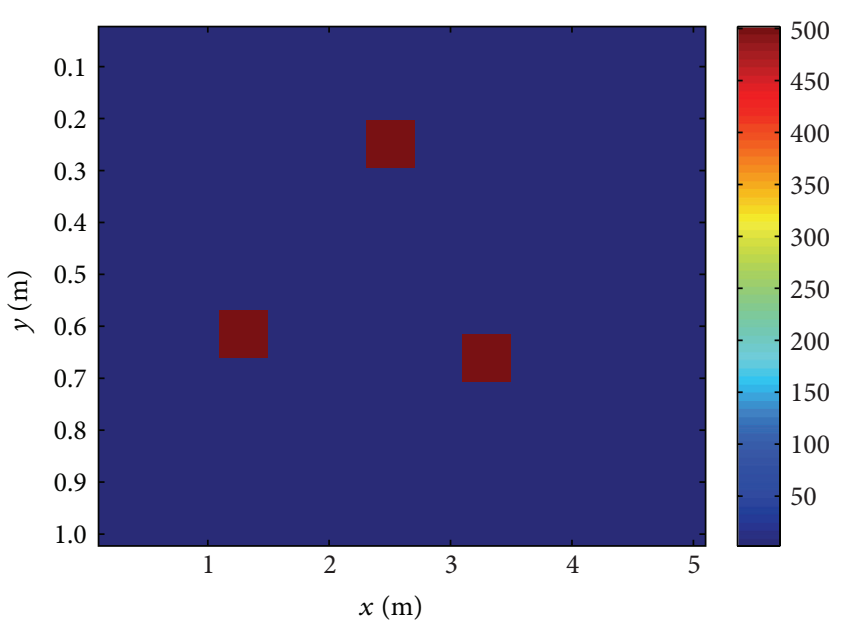

FIGURE 13: Original model.

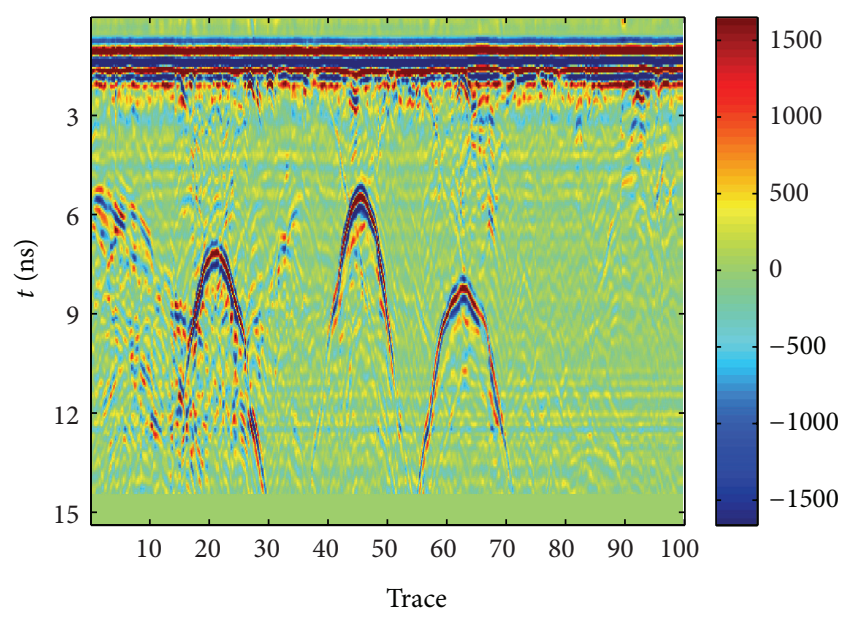

Figure 14: Practice measured data.

4.6. Practice Data Inversion. The practice measured data is provided by Wuhan Changjiang Engineering Geophysical Exploration \& Testing Co., Ltd.China. The testing object is a concrete member with three circle rebar in it. The centre of circle is $1.4 \mathrm{~m}, 0.6 \mathrm{~m} ; 2.2 \mathrm{~m}, 0.4 \mathrm{~m} ; 3.4 \mathrm{~m}, 0.7 \mathrm{~m}$, respectively, radius is $0.1 \mathrm{~m}$. The original model structure scheme is shown in Figure 13. Practice measured data is shown in Figure 14. Inversion result is shown in Figure 15. The relative error is $15.75 \%$. Before the inversion, it is necessary to preprocess the data because practice measured data has large noise.

\section{Conclusion}

This paper has addressed the perturbation homotopy method for solving inverse electromagnetic problem. Perturbation homotopy coupled with damping Gauss-Newton methods has been used in the experiments. Results show that when initial value is far away from true model, local convergent Gauss-Newton method just roughly gives the information of location and scale; the quality has huge error with true

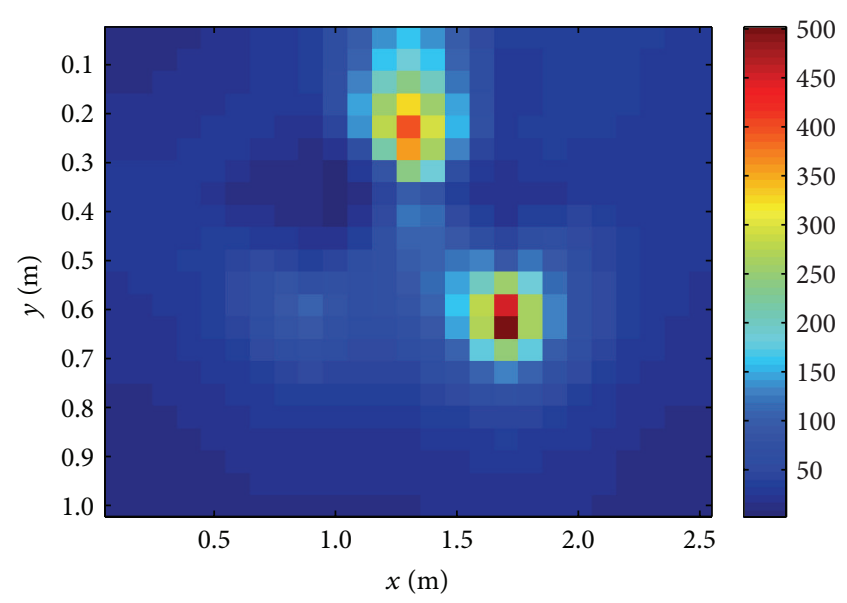

FIGURE 15: Inversion results.

model. Perturbation homotopy method is a widely convergent algorithm; it could give a more accurate inversion results even if initial value is far away from the original value. When adding Gauss white noise to measured data, we can see that, with noise decrease, numerical solution is convergence to original model, so numerical results support that our method is stable. Traditional homotopy theory tells us that optimum solution is determined at $\lambda=1$, but numerical results show that the solution will diverge when $\lambda$ is close to 1 because of regularization parameter impact. In a word, this method has clear advantages over damping Gauss-Newton method; it is an effective method, especially on aspects of wide convergence, computational efficiency, and precision and has broad application prospects.

\section{Conflict of Interests}

The authors declare that there is no conflict of interests regarding the publication of this paper.

\section{Acknowledgments}

This paper is supported by Natural Science Foundation of China, Project no. 41304093, the Fundamental Research Funds for the Central Universities, Project no. DL12BB20, and the Educational Commission of Heilongjiang Province of China no. 12533013.

\section{References}

[1] D. Colton and R. Kress, Inverse Acoustic and Electromagnetic Scattering Theory, Springer, New York, NY, USA, 1998.

[2] P. A. Eaton, "3-D electromagnetic inversion using integral equations," Geophysical Prospecting, vol. 37, pp. 407-426, 1989.

[3] J. Nocedal and S. Wright, Numerical Optimization, Springer, New York, NY, USA, 1999.

[4] G. A. Newman, S. Recher, B. Tezkan, and F. M. Neubauer, "3D inversion of a scalar radio magnetotelluric field data set," Geophysics, vol. 68, no. 3, pp. 791-802, 2003. 
[5] E. Haber, "Quasi-Newton methods for large-scale electromagnetic inverse problems," Inverse Problems, vol. 21, no. 1, pp. 305323, 2005.

[6] M. S. Zhdanov, S. Fang, and G. Hursan, "Electromagnetic inversion using quasi-linear approximation," Geophysics, vol. 65, no. 5, pp. 1501-1513, 2000.

[7] C. Torres-Verdín and T. M. Habashy, "Rapid numerical simulation of axisymmetric single-well induction data using the extended Born approximation," Radio Science, vol. 36, no. 6, pp. 1287-1306, 2001.

[8] H.-W. Tseng, K. H. Lee, and A. Becker, "3D interpretation of electromagnetic data using a modified extended Born approximation," Geophysics, vol. 68, no. 1, pp. 127-137, 2003.

[9] Z. Zhang, "3D resistivity mapping of airborne EM data," Geophysics, vol. 68, no. 6, pp. 1896-1905, 2003.

[10] M. S. Zhdanov, Geophysical Inverse Theory and Regularization Problems, Elsevier, Amsterdam, The Netherlands, 2002.

[11] M. S. Zhdanov, Geophysical Electromagnetic Theory and Methods, Elsevier, Amsterdam, The Netherlands, 2009.

[12] H. B. Han Bo, K. Z. Kuang Zheng, and L. J.-Q. Liu Jia-Qi, “A monotonous homotopy method for solving the resistivities of the earth," Acta Geophysica Sinica, vol. 34, no. 4, pp. 517-522, 1991.

[13] H. S. Fu, B. Han, and G. Q. Gai, "A wavelet multiscale-homotopy method for the inverse problem of two-dimensional acoustic wave equation," Applied Mathematics and Computation, vol. 190, no. 1, pp. 576-582, 2007.

[14] Y. He and B. Han, "A wavelet adaptive-homotopy method for inverse problem in the fluid-saturated porous media," Applied Mathematics and Computation, vol. 208, no. 1, pp. 189-196, 2009.

[15] D. W. Vasco, "Singularity and branching: a path-following formalism for geophysical inverse problems," Geophysical Journal International, vol. 119, no. 3, pp. 809-830, 1994.

[16] D. M. Dunlavy, D. P. O’Leary, D. Klimov, and D. Thirumalai, "HOPE: a homotopy optimization method for protein structure prediction," Journal of Computational Biology, vol. 12, no. 10, pp. 1275-1288, 2005. 


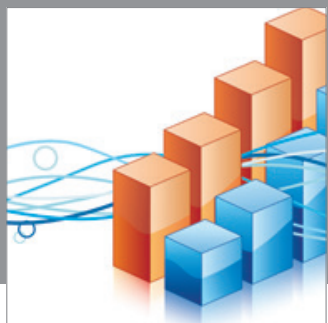

Advances in

Operations Research

mansans

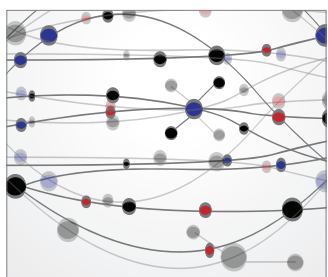

The Scientific World Journal
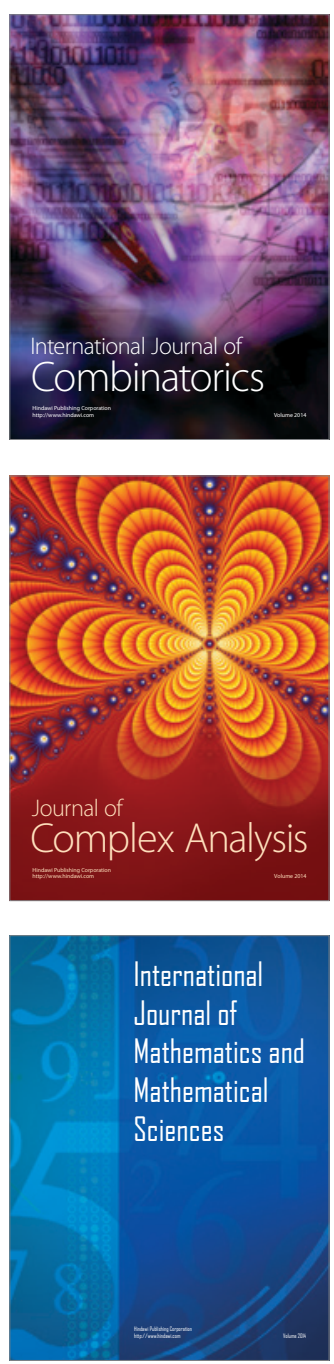
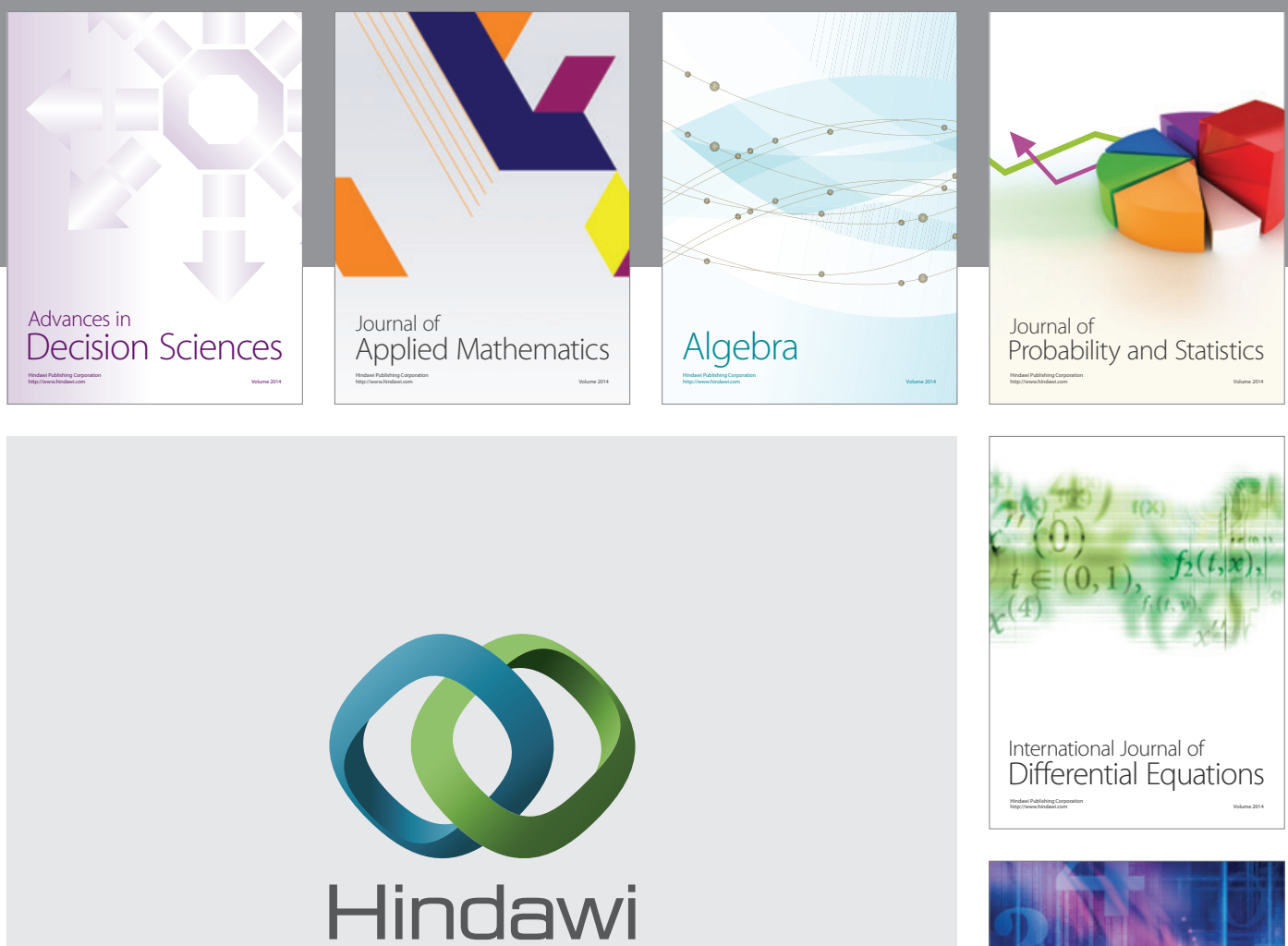

Submit your manuscripts at http://www.hindawi.com
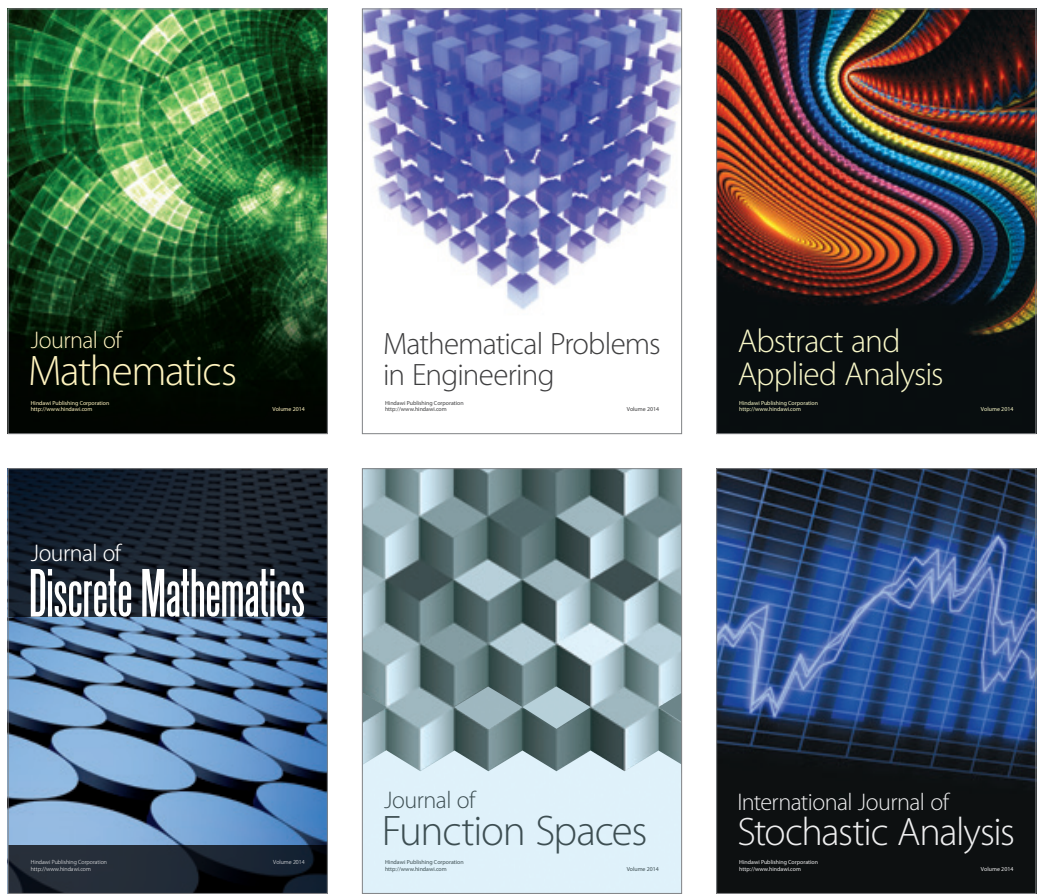

Journal of

Function Spaces

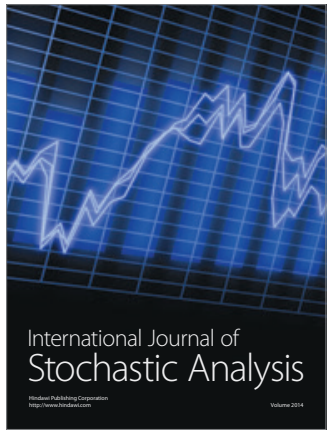

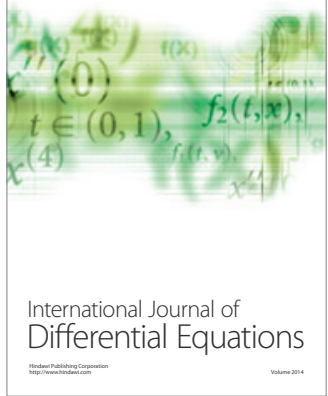
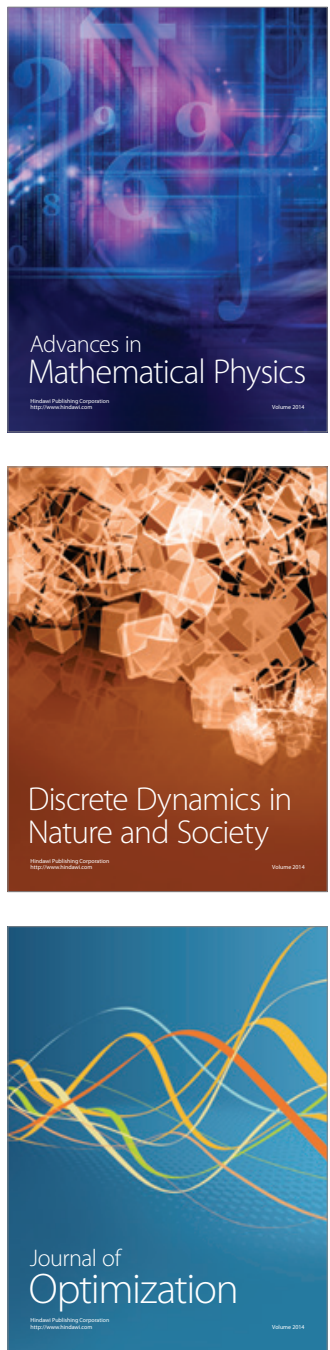